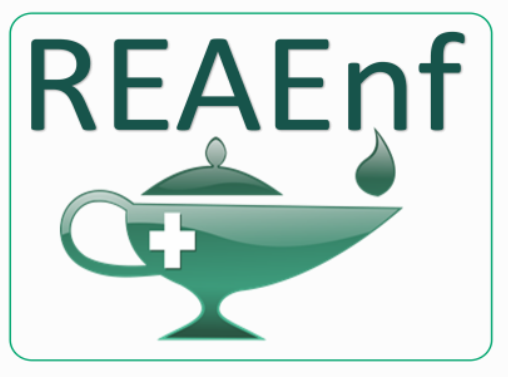

Revista Eletrônica Acervo Enfermagem
REVISÃO BILIOGRÁFICA

Recebido em: $12 / 2019$

Aceito em: 1/2020

Publicado em: 2/2020

\title{
Lesões precoces por pressão em pacientes com incontinência miccional e fecal em unidade de terapia intensiva
}

\author{
Press for press injuries in patients with micicional incontinence and close in intensive \\ therapy unit
}

Lesiones por presión temprana en pacientes con incontinencia miccional y fecal en una unidad de terapia intensiva

José Fabio da Silva ${ }^{1}$, Sandro Henrique de Holanda Carvalho ${ }^{1 *}$, Edinaldo Targino de Moura ${ }^{1}$, Igor Felipe Andrade Costa de Souza ${ }^{1}$.

\begin{abstract}
Resumo: A umidade causa nos pacientes com incontinência urinária e fecal um risco para o surgimento das úlceras por pressão, devido ao contato direto da urina e fezes com a pele. A etiologia da úlcera por pressão é predita pelas escalas de avaliação, mas para sua aplicação necessitam da habilidade do profissional enfermeiro. Este trabalho tem como objetivo revisar e compreender a relação da incontinência urinária e fecal com o surgimento precoce de lesões por pressão. Foi utilizado no estudo o método de revisão sistemática, constituído em cinco etapas, sendo eles o estabelecimento de critérios, categorização dos estudos e síntese do conhecimento produzido, analise dos dados e a interpretação e discussão dos resultados. Sugere-se dentre os estudos analisados que a incontinência urinária e/ou fecal está intimamente relacionada com lesões precoces por pressão. Uma das principais características dos pacientes críticos portadores de ulceras de decúbito é a presença da incontinência miccional e/ou fecal, no qual, mostra a importância da assistência de enfermagem no diagnóstico precoce dos riscos e prevenção destas lesões.
\end{abstract}

Palavras-chave: Úlcera por pressão, Incontinência urinária, Incontinência fecal.

\begin{abstract}
Moisture causes patients with urinary and fecal incontinence a risk for pressure ulcers due to direct contact of urine and feces with the skin. The etiology of pressure ulcer is predicted by the assessment scales, but for its application they require the skill of the professional nurse. This paper aims to review and understand the relationship between urinary and fecal incontinence and the early appearance of pressure injuries. The study used the systematic review method, consisting of five steps, which were the establishment of criteria, categorization of studies and synthesis of knowledge produced, data analysis and interpretation and discussion of results. It is suggested among the studies analyzed that urinary and / or fecal incontinence is closely related to early pressure injuries. One of the main characteristics of critically ill patients with decubitus ulcers is the presence of voiding and / or fecal incontinence, which shows the importance of nursing care in the early diagnosis of risks and prevention of these injuries.
\end{abstract}

Keywords: Pressure ulcer, Urinary incontinence, Fecal incontinence.

\footnotetext{
${ }^{1}$ Centro Universitário Maurício de Nassau, Caruaru - PE.

*E-mail: sandroenf23@gmail.com
} 
Resumen: La humedad causa a los pacientes con incontinencia urinaria y fecal un riesgo de úlceras por presión debido al contacto directo de orina y heces con la piel. Las escalas de evaluación predicen la etiología de la úlcera por presión, pero para su aplicación requieren la habilidad de la enfermera profesional. Este artículo tiene como objetivo revisar y comprender la relación entre la incontinencia urinaria y fecal y la aparición temprana de lesiones por presión. El estudio utilizó el método de revisión sistemática, que consta de cinco pasos, que fueron el establecimiento de criterios, la categorización de los estudios y la síntesis del conocimiento producido, el análisis de datos y la interpretación y discusión de los resultados. Se sugiere entre los estudios analizados que la incontinencia urinaria y / o fecal está estrechamente relacionada con lesiones tempranas por presión. Una de las características principales de los pacientes críticos con úlceras de decúbito es la presencia de micción y / o incontinencia fecal, lo que demuestra la importancia de la atención de enfermería en el diagnóstico temprano de riesgos y la prevención de estas lesiones.

Palabras clave: Úlcera por presión, Incontinencia urinaria, Incontinencia fecal.

\section{INTRODUÇÃO}

A Unidade de Terapia Intensiva (UTI) apesar de ser o local ideal para a intervenção com pacientes instáveis é considerada um ambiente hospitalar agressivo, tenso e traumatizante. Neste local se desenvolve um tratamento rigoroso e hostil para própria natureza, visto que, além da vulnerabilidade em que o paciente se encontra, existem fatores altamente prejudiciais à sua estrutura psicológica, como também fatores que favorecem o surgimento de complicações como úlceras de pressão e atrofias musculares (FERNANDES NCS, 2010).

Alguns enfermos que apresentam alteração da homeostase e risco de falência dos sistemas, são considerados "pacientes críticos" e, devido ao grau de comprometimento de seu estado clínico, necessitam de vigilância contínua da equipe de saúde. A úlcera por pressão constitui um fenômeno comum e uma das principais complicações de pessoas hospitalizadas em todo o mundo nos diferentes contextos da saúde, especialmente entre os hospitalizados nas unidades de terapia intensiva nos quais ela é uma ameaça adicional em doentes já comprometidos fisiologicamente (BORGHARDT AT, 2016; TEIXEIRA AFO, 2017).

A restrição prolongada ao leito provoca sintomas clínicos diversos e se traduzem em profundas alterações fisiológicas e bioquímicas afetando praticamente todos os órgãos e sistemas do corpo. As úlceras de decúbito são caracterizadas por ser uma lesão da pele, principalmente em locais de protuberâncias ósseas, que decorre de pressão associada a forças de atrito, por um período suficiente de tempo para causar isquemia tecidual. Os locais mais frequentes são: região sacra, trocanteres, calcâneos e proeminências ósseas. O desenvolvimento dessas lesões é multifatorial, sendo o principal fator a compressão exercida sobre um capilar, entre o arcabouço ósseo e uma superfície, colabando-o e ocasionando a necrose tissular, devido a períodos prolongados de isquemia e que reduzem a capacidade de recuperação tecidual (MATTIA AL, 2010; GOMES FF, 2010).

Os aspectos que predispõe a ocorrência dessas são classificados em primários, que abrange a pressão, o atrito e a fricção exercida sobre os tecidos ou secundários nos quais incluem o estado de mobilidade prejudicada, função sensório-motora prejudicada, desequilíbrio nutricional, idade avançada, alterações hematopoiéticas, diabetes, disfunção circulatória, medicamentos, questões psicossociais e a umidade, que compreende a incontinência fecal e urinária. (TEIXEIRA AFO, 2017).

Os pacientes com incontinência urinária e fecal, na qual é definida como uma condição que ocorre perda involuntária de urina ou de fezes encontra-se em risco para úlceras por pressão devido ao contato direto com a pele; a umidade mantida cria ambiente propício à proliferação bacteriana, além de permitir a ocorrência de fissuras na pele, originando feridas que, com a força exercida pela proeminência óssea, favorece o surgimento dessas lesões, e a progressão das mesmas (BORGHARDT AT, 2016; WADA A, 2010; CARVALHO MP, 2014). As escalas de avaliação apresentam o intuito de auxiliar os profissionais enfermeiros a identificar os riscos que um paciente tem de desenvolver úlcera por pressão. O Painel

REAEnf/EJNC | Vol.2 | e2591 | DOI: https://doi.org/10.25248/REAenf.e2591.2020 Página 2 de 11 
Consultivo Europeu sobre Úlcera por Pressão (EPUAP) recomenda que um instrumento de avaliação de risco inclua a avaliação geral e da pele, mobilidade, umidade, incontinência, nutrição e dor (BORGHARDT AT, 2016). Dentre as escalas mundiais, destacam-se a de Norton, Gosnell, Waterloo e Braden, sendo esta última a mais utilizada por ter sido submetida a diversos estudos e testes de confiabilidade.

A escala de Braden foi traduzida e validada para a população portuguesa em 2001 e consta de uma norma da Direção Geral da Saúde para a sua aplicação (ARAÚJO MT, 2011). Este instrumento apresenta 6 subescalas: percepção sensorial, umidade, atividade, mobilidade, nutrição, fricção e forças de deslizamento. Cada uma destas subescalas avalia o estado do doente, atribuindo-lhe uma classificação entre 1 e 4 (exceção do último que é de 1 a 3), comparando o estado do doente com o enunciado da escala e atribuindo o respectivo valor (BAVARESCO T, 2012).

A etiologia da úlcera por pressão é predita pelas escalas de avaliação, mas para sua aplicação necessitam da habilidade do profissional enfermeiro, sendo extremamente importante para o prognóstico dos pacientes com essa condição (CHACON JMF, 2013). As escalas de avaliação de úlcera por pressão predizem a etiologia, porém necessitam da embora requeira mais tempo na assistência para sua avaliação e acompanhamento.

As Úlceras por Pressão configuram um grave problema para os serviços de saúde, principalmente para as equipes de enfermagem, pois agravam o quadro clínico do paciente, podendo aumentar o risco de mortalidade e morbidade, e até mesmo acrescentar no tempo de internação do mesmo, além de elevar o custo do tratamento desenvolvido.

Diante da escassez de publicações abordando especificamente este tema, acreditamos ser de extrema importância o desenvolvimento de estudos relacionados a essa problemática, no intuito de se conhecer a intima relação entre a incontinência urinária e fecal e o surgimento precoce destas, tornando possível a adoção de medidas especializadas e direcionadas para a qualificação do atendimento a esses pacientes.

Diante da configuração do grave problema para os serviços públicos e do fato comum nos contextos de assistência à saúde, este trabalho teve por objetivo revisar e compreender a relação da incontinência urinária e fecal e o surgimento precoce de úlceras por pressão em pacientes na unidade de terapia intensiva.

\section{MÉTODOS}

Tratou-se de uma revisão sistemática da literatura, que tem como objetivo resumir toda a informação existente sobre um fenômeno de maneira imparcial e completa. Este trabalho foi constituído em cinco etapas, sendo elas seleção das hipóteses ou questões para a revisão, no qual o presente teve como questão norteadora Pacientes na unidade de terapia intensiva que apresentam incontinência urinária e/ou fecal possuem um surgimento precoce das lesões por pressão quando comparados com pacientes que não apresentam essas disfunções.

Foram considerados critérios de inclusão os artigos selecionados nas bases de dados Lilacs; Medline; Pubmed; Scholar e o Cochrane library, sendo que os descritores selecionados para busca dos artigos foram: Úlcera (ulcer), Incontinência urinária (urinary incontinence), Incontinência fecal (fecal incontinence), Umidade (moisture); artigos publicados entre o período de 2009 a 2019 e disponíveis nos idiomas português e inglês (Figura 1).

Foram excluídos os estudos que apresentavam lesão neurológica como causadora da incontinência urinária e/ou fecal, estudos que não correlacionavam o tema proposto com o surgimento da ulceração e a aparição de ulceras em pacientes hospitalizados (Figura 1).

Foi lido cada título e resumo de modo a confirmar se eles contemplavam a pergunta norteadora deste trabalho e se atendiam aos critérios de inclusão e exclusão estabelecida. Em seguida, foram analisados os dados e resultados através de uma síntese dos artigos (Quadro 1), reunindo o conhecimento e por fim a interpretação e discussão dos resultados. 
Figura 1 - Representação dos critérios de inclusão e exclusão.

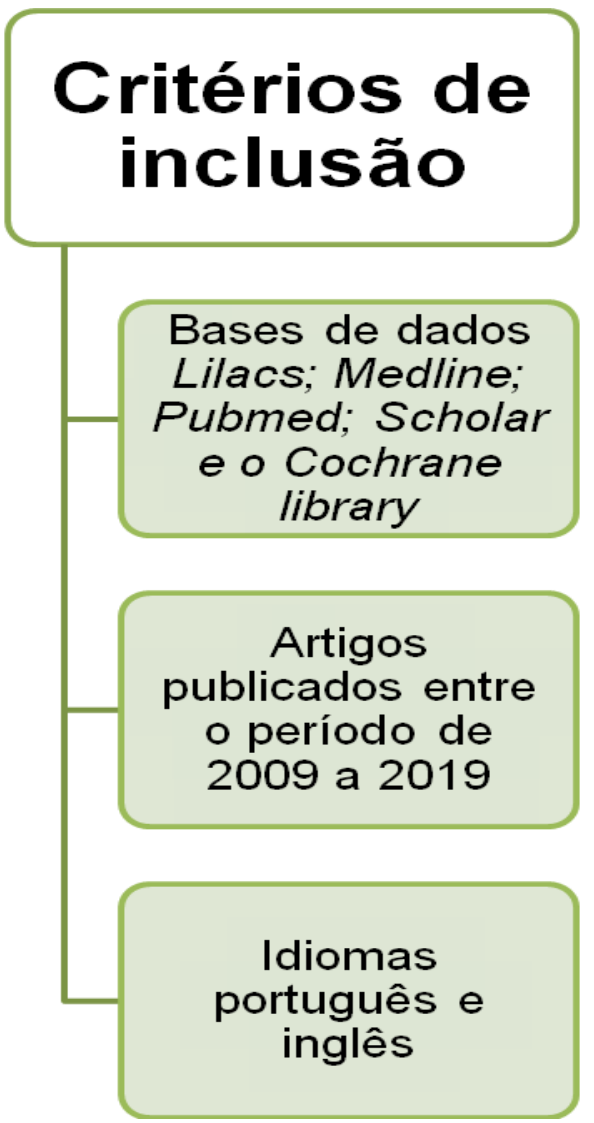

\section{Critérios de} exclusão

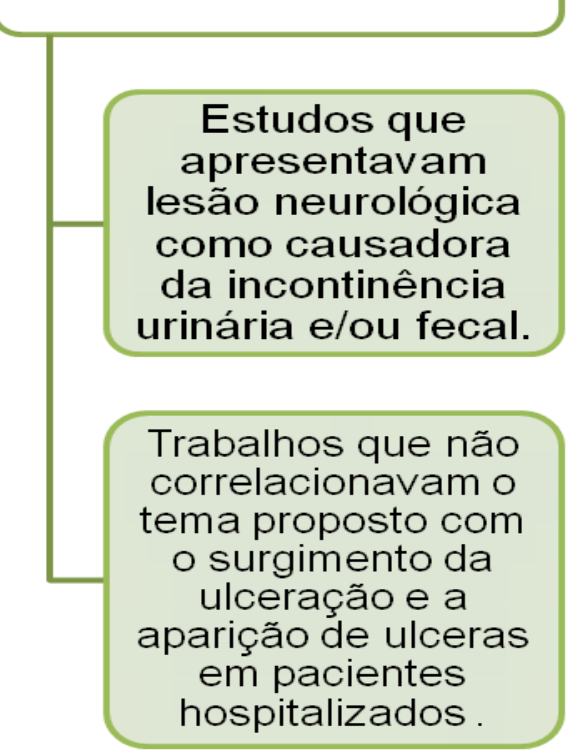

Fonte: Carvalho SHH, et. al., 2019. 
Quadro 1 - Artigos Selecionados

\begin{tabular}{|c|c|c|c|c|}
\hline AUTOR e ANO & IDIOMA & REVISTA & OBJETIVO & RESULTADOS \\
\hline $\begin{array}{l}\text { Anselmi MI, et. } \\
\text { al., } 2009\end{array}$ & Português & $\begin{array}{l}\text { Acta Paulista de } \\
\text { Enfermagem }\end{array}$ & $\begin{array}{l}\text { Estimar a ocorrência de úlcera por } \\
\text { pressão e descrever da ocorrência de } \\
\text { ações de enfermagem. }\end{array}$ & $\begin{array}{l}\text { São consideradas medidas preventivas, entre as áreas da } \\
\text { enfermagem, para o não desenvolvimento de ulceras por pressão, a } \\
\text { mobilização e o (re)posicionamento adequado do paciente; os } \\
\text { cuidados com a pele, utilização de creme hidratante; a indicação e o } \\
\text { monitoramento das condições nutricionais e de ingestão hídrica. }\end{array}$ \\
\hline $\begin{array}{l}\text { Araújo MT, et. } \\
\quad \text { al., } 2010\end{array}$ & Português & $\begin{array}{l}\text { Revista } \\
\text { Brasileira de } \\
\text { enfermagem }\end{array}$ & $\begin{array}{l}\text { Identificar os diagnósticos, de } \\
\text { enfermagem, em pacientes com risco } \\
\text { para úlcera por pressão em uma } \\
\text { Unidade de Terapia Intensiva de } \\
\text { Fortaleza - CE. }\end{array}$ & $\begin{array}{l}\text { Observou que especialmente as proeminências ósseas e fatores } \\
\text { nutricionais são os que apresentam relação direta com risco e } \\
\text { evolução das úlceras de pressão. }\end{array}$ \\
\hline $\begin{array}{l}\text { Borghardt AT, } \\
\text { et. al., } 2016\end{array}$ & Português & $\begin{array}{l}\text { Revista } \\
\text { Brasileira de } \\
\text { enfermagem }\end{array}$ & $\begin{array}{l}\text { Verificar a incidência de úlcera por } \\
\text { pressão e expor os fatores } \\
\text { associados ao desenvolvimento em } \\
\text { adultos internados nas unidades } \\
\text { intensivas de um hospital } \\
\text { universitário em Vitória, Espírito } \\
\text { Santo. }\end{array}$ & $\begin{array}{l}\text { Constatou-se elevada incidência da ulcera por pressão, na população } \\
\text { em estudo. }\end{array}$ \\
\hline $\begin{array}{l}\text { Chacon JMF, } \\
\text { et. al., } 2013\end{array}$ & Português & $\begin{array}{l}\text { Revista de } \\
\text { Saúde Coletiva }\end{array}$ & $\begin{array}{l}\text { Descrever o perfil dos pacientes, com } \\
\text { úlcera por pressão, da Unidade de } \\
\text { Terapia Intensiva de um Hospital de } \\
\text { São Paulo. }\end{array}$ & $\begin{array}{l}\text { Foram avaliados } 38 \text { pacientes em risco para o desenvolvimento de } \\
\text { lesões por pressão de acordo com a Escala de Braden e verificou em } \\
\text { seu estudo que a incontinência fecal foi identificada em } 73,7 \% \text { (28) } \\
\text { dos pacientes. }\end{array}$ \\
\hline $\begin{array}{l}\text { Creutzberg M, } \\
\text { et. al., } 2011\end{array}$ & Português & $\begin{array}{l}\text { Revista } \\
\text { enfermagem em } \\
\text { foco }\end{array}$ & $\begin{array}{l}\text { Constatar o risco de úlcera por } \\
\text { pressão em pacientes idosos } \\
\text { internados em Unidade de Terapia } \\
\text { Intensiva comparando as variáveis: } \\
\text { sexo, faixa etária e especialidade. }\end{array}$ & $\begin{array}{l}\text { A idade, associada à desnutrição, mobilidade e umidade são fatores } \\
\text { mais relevantes na fisiopatogênese das ulcerações. Onde o excesso } \\
\text { de umidade da pele, verificada nos resultados deste estudo, por } \\
\text { sudorese, incontinência urinária ou fecal, pode tornar o paciente mais } \\
\text { suscetível à maceração. }\end{array}$ \\
\hline $\begin{array}{l}\text { Crozeta K, et. } \\
\quad \text { al., } 2011\end{array}$ & Português & $\begin{array}{l}\text { Anais dos } 16+ \\
\text { senp } 2011\end{array}$ & $\begin{array}{l}\text { Investigar os fatores de risco para a } \\
\text { presença de úlceras por pressão, no } \\
\text { Hospital de Clínicas da Universidade } \\
\text { Federal do Paraná (HC/UFPR). }\end{array}$ & $\begin{array}{l}\text { Avaliou } 279 \text { pacientes e observou que no item continência, } 67,9 \% \text { dos } \\
\text { pacientes possuíam sonda vesical e incontinência fecal. }\end{array}$ \\
\hline
\end{tabular}

REAEnf/EJNC | Vol.2 | e2591 | DOI: https://doi.org/10.25248/REAenf.e2591.2020 Página 5 de 11 


\begin{tabular}{|c|c|c|c|c|}
\hline $\begin{array}{l}\text { Elias CMV, et. } \\
\quad \text { al., } 2014\end{array}$ & Português & $\begin{array}{c}\text { Revista } \\
\text { Interdisciplinar }\end{array}$ & $\begin{array}{l}\text { Analisar a produção de artigos } \\
\text { científicos, acerca da prevenção de } \\
\text { úlcera por pressão. }\end{array}$ & $\begin{array}{l}\text { A prevenção das ulceras por pressão é competência de toda a equipe } \\
\text { envolvida no cuidado e não responsabilidade única da equipe de } \\
\text { enfermagem. }\end{array}$ \\
\hline $\begin{array}{l}\text { Fernandes } \\
\text { NCS, Torres } \\
\text { GV., } 2010\end{array}$ & Português & $\begin{array}{l}\text { Revista Ciência } \\
\text { no Cuidado a } \\
\text { saúde. }\end{array}$ & $\begin{array}{l}\text { Verificar a associação entre os } \\
\text { fatores de risco na ocorrência de } \\
\text { ulcera por pressão em pacientes } \\
\text { internados na Unidade de Terapia } \\
\text { Intensiva. }\end{array}$ & $\begin{array}{l}\text { A multicausalidade no surgimento das ulceras por pressão remete a } \\
\text { refletir sobre a necessidade de uma avaliação clínica sistematizada do } \\
\text { paciente que contemple a complexidade dos fatores e condições } \\
\text { presentes durante a internação. }\end{array}$ \\
\hline $\begin{array}{l}\text { Furman GF, et. } \\
\text { al., } 2010\end{array}$ & Português & $\begin{array}{c}\text { Revista de } \\
\text { enfermagem }\end{array}$ & $\begin{array}{l}\text { Identificar a incidência de úlcera por } \\
\text { pressão e seus fatores de risco, em } \\
\text { internados nas Clínicas Cirúrgicas de } \\
\text { um Hospital Escola. }\end{array}$ & $\begin{array}{l}\text { Analisou } 36 \text { pacientes, sendo } 20 \text { com ulcera por pressão, através da } \\
\text { escala de Braden, e conseguiu verificar que todos pacientes com } \\
\text { úlcera de pressão, realizavam as eliminações em fralda. }\end{array}$ \\
\hline $\begin{array}{l}\text { Loureiro CA, } \\
\text { Laranjeira ES., } \\
\quad 2017\end{array}$ & $\begin{array}{l}\text { Português } \\
\text { Europeu }\end{array}$ & $\begin{array}{c}\text { Revista Saúde } \\
\text { pública }\end{array}$ & $\begin{array}{l}\text { Apontar e descrever os fatores de } \\
\text { risco das úlceras de pressão em } \\
\text { doentes, internados num Hospital } \\
\text { Central Português. }\end{array}$ & $\begin{array}{l}\text { Analisou } 30 \text { pacientes com úlceras por pressão através da escala de } \\
\text { Braden, e verificou que a incontinência estava presente em } 82,4 \% \\
\text { dos pacientes, sendo que apenas } 2,9 \% \text { apresentavam incontinência } \\
\text { urinária, } 73,5 \% \text { incontinência urinária e fecal. }\end{array}$ \\
\hline $\begin{array}{l}\text { Luz SR, et. al., } \\
2010\end{array}$ & Português & $\begin{array}{l}\text { Revista de } \\
\text { geriatria e } \\
\text { gerontologia }\end{array}$ & $\begin{array}{l}\text { Apresentar e discutir as medidas que } \\
\text { visam à prevenção das úlceras de } \\
\text { pressão. }\end{array}$ & $\begin{array}{l}\text { Através de uma revisão bibliográfica analisou } 28 \text { artigos científicos e } 8 \\
\text { livros técnicos e observou que a incontinência urinária e/ou fecal são } \\
\text { fatores intimamente relacionados com a formação de úlceras de } \\
\text { pressão. }\end{array}$ \\
\hline $\begin{array}{c}\text { Manderlier B, } \\
\text { et. al., } 2019\end{array}$ & Inglês & J. Adv. Nurs. & $\begin{array}{l}\text { Explorar quais fatores são } \\
\text { associados à presença de úlceras } \\
\text { por pressão da categoria I-IV nos } \\
\text { locais mais vulneráveis. }\end{array}$ & $\begin{array}{l}\text { A incontinência e a umidade são importantes fatores na avaliação do } \\
\text { risco de úlcera por pressão e que está significativamente associada às } \\
\text { úlceras por pressão sacral da categoria I-IV. }\end{array}$ \\
\hline $\begin{array}{l}\text { Mattias AL, et. } \\
\text { al., } 2010\end{array}$ & Português & $\begin{array}{c}\text { Revista Saúde } \\
\text { Coletiva }\end{array}$ & $\begin{array}{l}\text { Identificar os fatores de risco e as } \\
\text { medidas preventivas, para úlcera por } \\
\text { pressão, de uma unidade de terapia } \\
\text { intensiva adulto, do município de } \\
\text { Santos. }\end{array}$ & $\begin{array}{l}\text { A amostra foi constituída de } 30 \text { pacientes e após a coleta de dados } \\
\text { Mattia expões que com relação aos fatores de risco secundários, } 13 \\
(43,4 \%) \text { pacientes com idade entre } 60 \text { a } 75 \text { anos, com incontinência } \\
\text { urinária e uso de fralda descartável, apresentaram ulceras de pressão. }\end{array}$ \\
\hline
\end{tabular}

REAEnf/EJNC | Vol.2 | e2591 | DOI: https://doi.org/10.25248/REAenf.e2591.2020 Página 6 de 11 


\begin{tabular}{|c|c|c|c|c|}
\hline $\begin{array}{l}\text { Menegon DB, } \\
\text { et. al., } 2011\end{array}$ & Português & $\begin{array}{l}\text { Revista texto } \\
\text { contexto } \\
\text { enfermagem. }\end{array}$ & $\begin{array}{l}\text { Identificar a pontuação das } \\
\text { subescalas que avaliam o risco para } \\
\text { úlcera por pressão na Escala de } \\
\text { Braden e associá-las aos motivos de } \\
\text { internação hospitalar, às } \\
\text { comorbidades e às características } \\
\text { demográficas de pacientes adultos. }\end{array}$ & $\begin{array}{l}\text { A utilização do escore total apresentado pela Escala de Braden } \\
\text { fornece uma visão geral do paciente, melhorando o direcionamento do } \\
\text { cuidado necessário à prevenção da ulcera por pressão. }\end{array}$ \\
\hline $\begin{array}{l}\text { Patente MEF, } \\
\text { et. al., } 2011\end{array}$ & Português & $\begin{array}{l}\text { Revista } \\
\text { Percurso } \\
\text { acadêmico }\end{array}$ & $\begin{array}{l}\text { Avaliar a ocorrência de úlceras por } \\
\text { pressão e suas características em } \\
\text { pacientes internados em uma } \\
\text { Unidade de Terapia Intensiva no } \\
\text { interior de Minas Gerais. }\end{array}$ & $\begin{array}{l}\text { Foram examinados os prontuários de } 7 \text { indivíduos que apresentavam } \\
\text { ulcerações e concluíram que a incontinência urinária não foi um fator } \\
\text { predisponente à ocorrência de lesões cutâneas. }\end{array}$ \\
\hline $\begin{array}{l}\text { Salomé MG., } \\
2010\end{array}$ & Português & $\begin{array}{l}\text { Revista Saúde } \\
\text { Coletiva }\end{array}$ & $\begin{array}{l}\text { Descrever os pacientes com úlcera } \\
\text { por pressão, internados em uma } \\
\text { Unidade de Terapia Intensiva adulto. }\end{array}$ & $\begin{array}{l}\text { Foram avaliados } 31 \text { prontuários, concluindo que a úlcera por pressão } \\
\text { estava presente em } 31,25 \% \text { dos pacientes, onde } 30 \quad(17,54 \%) \\
\text { apresentaram o fator incontinência urinaria/fecal. }\end{array}$ \\
\hline $\begin{array}{l}\text { Maurício AB, } \\
\text { et. al., } 2014\end{array}$ & Português & $\begin{array}{l}\text { Revista de } \\
\text { enfermagem }\end{array}$ & $\begin{array}{l}\text { Entender o conhecimento sobre } \\
\text { úlceras por pressão, dos profissionais } \\
\text { de enfermagem, do Centro de } \\
\text { Terapia Semi-intensiva de um } \\
\text { hospital universitário. }\end{array}$ & $\begin{array}{l}\text { Os profissionais apresentaram conhecimento insatisfatório sobre } \\
\text { ulcera por pressão, o que demonstra necessidade de atualização e } \\
\text { capacitação. }\end{array}$ \\
\hline $\begin{array}{l}\text { Sousa RG, et. } \\
\text { al., } 2016\end{array}$ & Português & $\begin{array}{l}\text { Revista } \\
\text { Universidades } \\
\text { Ciências da } \\
\text { Saúde } \\
\end{array}$ & $\begin{array}{l}\text { Descrever os fatores de risco e o } \\
\text { perfil clínico de pacientes críticos } \\
\text { portadores de úlcera por pressão. }\end{array}$ & $\begin{array}{l}\text { Analisou } 15 \text { publicações e ao analisar pode perceber que as lesões } \\
\text { provocadas pela umidade tornam a pele suscetível a lesões por } \\
\text { fricção. }\end{array}$ \\
\hline $\begin{array}{l}\text { Teixeira AFO, } \\
2017\end{array}$ & Português & $\begin{array}{l}\text { Revista } \\
\text { Integrativa de } \\
\text { literatura }\end{array}$ & $\begin{array}{l}\text { Reunir estudos que compararam as } \\
\text { melhores condutas para prevenção } \\
\text { das ulceras por pressão. }\end{array}$ & $\begin{array}{l}\text { A posição prona mostrou a reabertura de unidades alveolares não } \\
\text { ventiladas, melhorando a relação ventilação/perfusão em pacientes } \\
\text { submetidos à ventilação mecânica invasiva. }\end{array}$ \\
\hline $\begin{array}{l}\text { Wada A, et. al., } \\
\qquad 2010\end{array}$ & Português & $\begin{array}{l}\text { Revista de } \\
\text { Medicina }\end{array}$ & $\begin{array}{l}\text { Análise dos fatores de risco para } \\
\text { úlceras por pressão e a eficácia do } \\
\text { tratamento com pressão negativa. }\end{array}$ & $\begin{array}{l}\text { As ulceras por pressão com complicações avançadas, o tratamento } \\
\text { determinante na sua resolução é o cirúrgico. }\end{array}$ \\
\hline
\end{tabular}

Fonte: Silva, JF, et al., 2019. 


\section{RESULTADOS}

Foram encontradas 44 referências nas bases de dados. Selecionou-se ao todo 20 artigos, pois contemplavam a pergunta norteadora e atendiam aos critérios previamente estabelecidos, nesta revisão.

Salomé GM (2005) em uma abordagem quantitativa, durante o mês de outubro do ano de 2005, avaliou 31 prontuários de pacientes internados na Unidade de Terapia Intensiva (UTI) adulto, do Hospital Geral de Vila Nova Cachoeirinha, localizado na zona norte da cidade de São Paulo, constatou que a incidência para úlcera por pressão foi de $31,25 \%$, onde $30(17,54 \%)$ dos pacientes observados apresentaram o fator incontinência urinaria/fecal.

Mattia AL, et al. (2007) realizaram um estudo de campo, descritivo, transversal, com abordagem quantitativa, em um hospital não governamental que atende pacientes cadastrados no Sistema Único de Saúde (SUS), particulares e de convênios de saúde, localizado no município de Santos, estado de São Paulo, Brasil. A amostra deste estudo foi do tipo não probabilística, constituída por 30 pacientes com 18 anos de idade ou mais, internados na unidade e para obtenção dos dados utilizou-se um instrumento que abrangia os aspectos sócio-demográficos e clínicos dos pacientes, os fatores de risco e medidas de prevenção para ocorrência das lesões e classificação das mesmas quando presentes. Após a coleta de dados Mattia expões que com relação aos fatores de risco secundários, $13(43,4 \%)$ pacientes com idade entre 60 a 75 anos, com incontinência urinária e uso de fralda descartável, apresentaram ulceras de pressão.

Sales MCM, et al. (2008) através de um estudo transversal com 16 pacientes, desenvolvido em uma unidade de clínica médico-cirúrgica de um hospital universitário de Belo Horizonte - MG., utilizou um questionário sociodemográfico, epidemiológico e a escala de Braden para avaliação dos fatores de risco, localização anatômica e avaliação clínica da úlcera, e pode observar que a umidade, a imobilidade e o cisalhamento estiveram presentes em todos os casos de ulceras, mesmo que em níveis variados. $E$ constatou, também, que a umidade excessiva, provocada por incontinência urinária, ou fecal, pode ser evitada diminuindo o intervalo de troca de roupas e aumentando o rigor na higienização e secagem após o banho do paciente.

Anselmi ML, et al. (2009) complementaram o que Sales MCM, et. al. (2008) descreveram em seu estudo, em 2008. Que são consideradas medidas preventivas, entre as áreas da enfermagem, para o não desenvolvimento de ulceras por pressão, a mobilização e o (re)posicionamento adequado do paciente; os cuidados com a pele por meio de uso de técnicas apropriadas de higiene, utilização de creme hidratante; a indicação e o monitoramento das condições nutricionais e de ingestão hídrica.

Furman GF, et al. (2009) desenvolveu uma pesquisa entre os meses de novembro e dezembro de 2008 a janeiro de 2009 nas Unidades de Internação Feminina e Masculina do Hospital Universitário Regional do Norte do Paraná (HURNP), com 36 pacientes sendo 20 com ulcerações que possuíam grau de dependência três ou quatro e atingiram um escore de até 13 como resultado da aplicação da Escala de Braden e com isso conseguiram verificar que todos pacientes com úlcera de pressão, realizavam as eliminações em fralda. A excreção urinária era espontânea em fralda para 35\% (7) e os demais $13(65 \%)$ utilizavam sonda vesical de demora, dispositivo urinário, cistostomia ou sondagem vesical de alívio.

Creutzberg M, et al. (2010) realizou uma pesquisa exploratória analítica e retrospectiva, de janeiro a julho de 2010, com 216 pacientes internados na UTI de um hospital universitário do Rio Grande do Sul, inseridos no Protocolo de Prevenção de Úlceras de Pressão, o autor os separou em adultos jovens (22-39 anos), médios (40-59) e idosos (60 e mais), em que foram avaliados por meio da escala de Braden, chegando ao resultado que a idade, associada à desnutrição, mobilidade e umidade são fatores mais relevantes na fisiopatogênese das ulcerações. Onde o excesso de umidade da pele, verificada nos resultados deste estudo, por sudorese, incontinência urinária ou fecal, pode tornar o paciente mais suscetível à maceração.

Chacon F, et al. (2010) realizaram um estudo transversal e analítico, de junho a agosto de 2010, desenvolvido na Unidade de Terapia Intensiva do Pronto Socorro do Hospital São Paulo (HSP) no Município de São Paulo, no qual foram avaliados 38 pacientes em risco para o desenvolvimento de lesões por 
pressão de acordo com a Escala de Braden e verificou em seu estudo que a incontinência fecal foi identificada em 73,7\% (28) dos pacientes. Menegon DB, et al. (2012) relatou que o primeiro passo na prevenção dessas lesões é a avaliação de risco, com um instrumento medidor de risco, pois permite aos enfermeiros reunir informações que identifique os pacientes com maior vulnerabilidade e, a partir disto, planejar os cuidados mais adequados.

Bavaresco T (2011) de março de 2010 a dezembro de 2011 analisou 30 enfermeiros, de dois hospitais universitários de grande porte, um da região Sul e outro da região Sudeste do Brasil, com base nas 51 intervenções descritas pela Classificação de intervenções de enfermagem (NIC), que apresenta como foco as intervenções do enfermeiro na execução de sua prática profissional, as quais são formadas por uma lista de atividades que irão tratar os fatores de risco e as características definidoras (sinais e sintomas) de um diagnóstico estabelecido. As intervenções da NIC incluem tanto cuidados diretos, ou seja, tratamentos realizados por meio da interação com o paciente, os quais incluem ações no âmbito fisiológico e psicossocial, bem como, as ações práticas, de apoio e aconselhamento para a vida. Já os cuidados indiretos se constituem de tratamentos ao paciente realizados a distância, com ações voltadas ao gerenciamento do ambiente. Estas intervenções estão inseridas dentro de um domínio e de uma classe e são compostas por título, definição e um código numérico padrão submetidas à validação de conteúdo. $\mathrm{E}$ através disso puderam concluir que existem quatro dessas intervenções que estão localizadas no domínio Fisiológico complexo, que são: tratamentos tópicos, Monitorização de Sinais Vitais, Cuidados na Incontinência Urinária, Posicionamento e Terapia Nutricional, consideradas aplicáveis ao cuidado de pacientes em risco de úlceras por pressão.

Sousa RG, et al. (2011) selecionou o método revisão integrativa da literatura, a qual permite a síntese de estudos publicados, possibilitando conclusões gerais a respeito de um estudo particular da área e analisou 15 publicações e ao analisar todos os estudos pode perceber que as lesões provocadas pela umidade tornam a pele suscetível a lesões por fricção, sendo frequente quando há associação entre incontinência fecal e urinária concomitante.

A Escala de Braden foi desenvolvida para atenuar a incidência de lesões por pressão, sendo composta por seis subescalas: percepção sensorial, umidade da pele, atividade, mobilidade, estado nutricional, friç̧ão e cisalhamento. Esta escala categoriza a umidade em Completamente molhado, muito molhado, ocasionalmente molhado e raramente molhado fazendo com que o enfermeiro conheça verdadeiramente o quanto o paciente esta exposto ao fator de risco umidade, confirmando assim seu uso nos estudos (FURMAN GF, 2009; CREUTZBERG M, 2010; CHACON F, 2010).

De maneira oposta aos autores citados acima, Araújo TM, et al. (2011) através de um estudo transversal, utilizou a escala de Waterlow para avaliar os riscos de úlcera por pressão, em um Hospital do Município de Fortaleza-CE, que consistiu na avaliação de 10 itens: sexo/idade; índice de massa corporal (IMC); tipo de pele, continência, mobilidade, subnutrição do tecido celular, deficiência neurológica, cirurgia de grande porte/trauma, apetite, medicação e sonda nasogástrica (SNG) ou sonda nasoenteral (SNE), cada item possuí valores que variam entre 0 e 5 . Foi utilizada também a sistematização da assistência de enfermagem, com a realização do histórico de enfermagem e exame físico para levantamento dos diagnósticos de enfermagem nos pacientes com risco para essas lesões, observando que especialmente as proeminências ósseas e fatores nutricionais são os que apresentam relação direta com risco e evolução das úlceras de pressão.

Crozeta K, et al. (2009) de abril a maio de 2009, utillizou a escala de Waterlow para avaliar 279 pacientes em unidades de internação do HC/UFPR de Curitiba, e observou diferentemente de Araújo que no item continência, $67,9 \%$ dos pacientes possuíam sonda vesical e incontinência fecal. Nesta revisão de literatura se pode observar que estudos que utilizaram a escala de avaliação Waterlow chegaram à conclusão que a incontinência urinária e fecal não apresenta influencia direta no surgimento precoce de ulceras por pressão, apesar de essas duas escalas apresentarem o item Continência e Umidade, respectivamente. Em um estudo, realizado por Laranjeira CA e Loureiro S (2011) de 1 a 30 de Junho de 2011, analisou 30 pacientes com úlceras por pressão, internados no hospital Garcia da Orta, Instituição 
central de Lisboa e Vale do Tejo - Portugal verificou através da escala de Braden e de uma entrevista sociodemográfica que a incontinência estava presente em $82,4 \%$ dos pacientes, sendo que apenas $2,9 \%$ apresentavam incontinência urinária, 73,5\% incontinência urinária e fecal.

Luz SR, et al. (2011) através de uma revisão bibliográfica analisou 28 artigos científicos e 8 livros técnicos e observou que a incontinência urinária e/ou fecal são fatores intimamente relacionados com a formação de úlceras de pressão, sugerindo que pacientes incontinentes apresentam risco de até cinco vezes maior, quando comparados a pacientes não incontinentes.

Patente MEF, et al. (2011) realizou um estudo de caso com pacientes internados em uma Unidade de Terapia Intensiva Adulto de um hospital do interior de Minas Gerais, foram examinados os prontuários de 7 indivíduos que apresentavam ulcerações, as informações coletadas foram sexo, idade, tempo de internação, uso e tempo de sedação, presença de traqueostomia/intubação, tipo de dieta recebida, presença de incontinência vesical e fecal e concluíram que a incontinência urinária não foi um fator predisponente à ocorrência de lesões cutâneas; porém, quatro dos sete pacientes que evoluíram com ulcera, desenvolveram incontinência fecal.

Manderlier B, et al. (2019) analisaram transversalmente os pacientes de todas as instituições de saúde da Holanda, com o objetivo de explorar quais fatores são associados à presença de úlceras por pressão da categoria I-IV nos locais mais vulneráveis e concluíram que a incontinência e a umidade são importantes fatores na avaliação do risco de úlcera por pressão e que está significativamente associada às úlceras por pressão sacral da categoria I-IV.

\section{CONSIDERAÇÕES FINAIS}

Diante desta revisão literária, foi possível observar que uma das principais características dos pacientes críticos portadores de ulceras por pressão é a presença de incontinência miccional e fecal, no qual, mostra a importância da assistência de enfermagem na prevenção destas lesões, principalmente nas Unidades de Terapia Intensiva, onde esse problema é prevalente.

\section{REFERÊNCIAS}

1. ANSELMI ML, et. al. Incidência de ulcera por pressão e ações de enfermagem. Acta Paulista de Enfermagem, 2009; 23: 257-264.

2. ARAUJO MT, et. al. Diagnósticos de enfermagem para pacientes em risco de desenvolver úlcera por pressão. Revista Brasileira de Enfermagem, 2011; 64: 671-676.

3. BORGHARDT AT, et. al. Úlcera por pressão em pacientes críticos: incidência e fatores associados. Revista brasileira de enfermagem, 2016; 69: 460-470.

4. CHACON JMF, et. al. Aspectos epidemiológicos da paciente com pressão arterial na unidade de terapia intensiva do pronto-socorro de um hospital de ensino de São Paulo. Revista de Saúde Coletiva, 2013; 10: 14-19.

5. CREUTZBERG M, et. al. Fatores de risco para pressão arterial em idosos de Unidade de Terapia Intensiva. Revista enfermagem em foco, jan./mar. 2011; 2: 133-136.

6. CROZETA K, et. al. Fatores de risco para presença de úlcera por pressão: aplicação da escala de waterlow. $16+$ senp 2011, 2011; 201: 1059-1062.

7. ELIAS CMV, et. al. Compilação de evidências científicas sobre a prevenção da úlcera por pressão. Revista interdisciplinar, 2014; 7: 183-192.

8. FERNANDES NCS, et. al. Fatores de risco e condições predisponentes para úlcera de pressão em pacientes de terapia intensiva. Revista Eletrônica de Enfermagem, 2010; 10: 733-746.

9. FURMAN GF, et. al. Úlceras por pressão: incidência e associação de fatores de risco em pacientes de um hospital universitário. Revista de enfermagem, 2010; 4: 1506-1514.

10. GOMES FF. Úlceras por pressão: incidência e associação de fatores de risco em pacientes de um hospital universitário. Revista de enfermagem, 2010; 4(3): 1506-1514.

11. LOUREIRO CA, LARANJEIRA ES. Fatores de risco das úlceras de pressão em pacientes internados num hospital. Revista Saúde pública, 2017; 19(1): 99-104.

12. LUZ SR, et. al. Úlceras de pressão. Revista de geriatria e gerontologia, 2010; 4: 36-43. 
13. MANDERLIER B, et. al. Modifiable patient -related factors associated with pressure ulcers on the sacrum and heels: secondary data analyses. J Adv Nurs, 2019; 7: 01-38.

14. MATTIA AL, et. al. Úlcera por Pressão em UTI: fatores de risco e medidas de prevenção. Revista Saúde coletiva, 2010; 7: 296- 299.

15. MAURICIO AB, et. al. Conhecimentos dos profissionais de enfermagem relacionados às úlceras por pressão. Revista de enfermagem da UFSM, 2014; 4: 751-760.

16. MENEGON DB, et. al. Analise das subescalas de Braden como indicadores de risco para a úlcera por pressão. Texto Contexto Enfermagem, 2011; 21: 854-861.

17. PATENTE MEF, et. al. Úlcera por pressão em pacientes internados em terapia de terapia intensiva. Percurso acadêmico, 2011; 1: 51-60.

18. SALOMÉ MG. Ocorrência de úlcera por pressão em indivíduos internados em uma unidade de Terapia Intensiva. Revista Saúde coletiva, 2010; 7(42): 183-188.

19. SOUSA RG, et. al. Fatores associados à úlcera por pressão (UPP) em pacientes críticos: revisão integrativa da literatura. Revista Universidades Ciências da Saúde, 2016; 14: 77-84.

20. TEIXEIRA AFO. Mudanças de decúbito em paciente crítico em ventilação mecânica invasiva: uma revisão integrativa de literatura. FASERRA, 2017.

21. WADA A, et. al. Úlceras por pressão. Revista de Medicina, 2010; 89: 170-177. 